\title{
Automatic Cell Nuclei Segmentation Using Superpixel and Clustering Methods in Histopathological Images
}

\author{
Gamze Mendi and Cafer Budak
}

\begin{abstract}
It is seen that there is an increase in cancer and cancer-related deaths day by day. Early diagnosis is vital for the early treatment of the cancerous area. Computer-aided programs allow for the early diagnosis of unhealthy cells that specialist pathologists diagnose due to efforts.

In this study, clustering and superpixel segmentation techniques were used to detect cell nuclei in high-resolution histopathology images automatically. As a result of the study, the successful performances of the segmentation algorithms were analyzed and evaluated. It is seen that better success is obtained in the Watershed and FCM algorithms in highresolution histopathological images used. Quickshift and SLIC methods gave better results in terms of precision. It is seen that there are k-Means and FCM algorithms that provide the best performance in $\mathrm{F}$ measure (F-M), and the correct negative rate (TNR) is more successful in Quickshift, kMeans, and SLIC methods.
\end{abstract}

Index Terms - Segmentation, histopathological image analysis, superpixels, image processing

\section{INTRODUCTION}

$\mathrm{C}$ ANCER, HAS been among the serious health problems in recent years, is among the world's top causes of death. Humans with different age groups and other characteristics are getting cancer and lose their life. When this situation is analyzed with Turkey's health statistics, approximately 96 thousand men and 67 thousand women get cancer in a year.

While lung and prostate cancers are common in men, one in four women are diagnosed with breast cancer compared to other cancers [1]. Early diagnosis of the tumor plays a crucial role in early treatment. Evolving visualization devices allow high-resolution histopathological images to allow laboratory examination that causes early treatment for cancer [2]. The development of high-resolution devices enables specialist pathologists to analyze texture with lesser

GAMZE MENDI, is with the Department of Electrical and Electronics Engineering University of Batman University, Batman, Turkey,(e-mail: gamzemendi2@gmail.com).

(iD) https://orcid.org/0000-0003-4295-0410

CAFER BUDAK is with the Department of Biomedical Engineering, Dicle University, Diyarbakir Turkey, (e-mail: cafer.budak@dicle.edu.tr).

(iD https://orcid.org/0000-0002-8470-4579 effort. It is seen that digital image processing, compared to microscopy, is advantageous. The fact that a certain number of people can observe with the microscope at the same time, and the specialized pathologist who repeatedly has no opportunity to examine this tissue, can be considered a disadvantage. With digital imaging devices, hundreds of specialist pathologists can read the tissue simultaneously [2]. Expert pathologists perform microscopic examinations of patients' tissues or organs for diagnosis. Before reviewing the biopsy tissue, they undergo it to certain stages.

The tissue taken undergoes preliminary processes such as fixation, staining, cutting, and scanning. When these processes are performed successfully, fat tissue, cytoplasm, and cellular structures become distinguishable. Making these procedures unhealthy prevents the differentiation of systems in the tissue. It reduces the success of segmentation in the computer-aided analysis due to destructive processes [3]. There are many studies of researchers related to this subject. Kayaaltı and Aksebzeci [4] studied a data set containing benign_and malignant tumor images in breast cancer. They perform the Gray Level Co-Occurrence Matrices feature extraction matrix. They achieved $82.06 \%$ success with the Random Forests classifier. Albayrak and Bilgin [5] used a deep learning-based SegNet method to divide cellular structures in high-resolution histopathological images. They observed that SegNet was quite successful in segmentation cellular networks compared to other methods (k-Means, Otsu, Irshad) that are frequently used.

Çakır vd. [6] designed a system that automatically detects cancer precursor lesions in their study using the convolutional neural networks method of precursor lesions in cervical cancer. The system achieved $92 \%$ success in distinguishing lesions from images. They created a model with an 81.71 percent booming segment according to the Dice coefficient. Turan and Bilgin [7] used histopathological images obtained as a result of hematoxylin and eosin staining of deep learning and cancer diagnosed biopsy samples on breast pathology images, and comparatively evaluated the semantic segmentation process using SegNet [8] and U-Net [9] algorithms, and they found that the average F-measure of SegNet was 0.73, the training sensitivity was $85.64 \%$, the average F-measure of U-Net was 0.73 , the training sensitivity was $72.87 \%$. They have achieved successful results in the $\mathrm{KNN}$, as long as the number of neighbors is below 10. They achieved a success rate of 0.99 in terms of precision, sensitivity, and f1-score. Many studies related to segmentation in the literature [11-14] Huaman et 
al. [15] suggest automatic identification of diseased leaf areas. The technique used the SLIC algorithm in zones called superpixels with a similar color to form a group. They used it to train neural networks to classify whether superpixels are healthy or unhealthy. They found the average F-score to be 0.67. Yuan et al. [16] developed a superpixel-based and boundary-sensitive convolutional neural network for superpixel-based liver disease zone automatic segmentation. They divided them into three classes as inner liver, liver border, and posterior liver. They obtained an SBBS-CNN with an average membrane similarity coefficient of $97.31 \pm$ $0.36 \%$ and an average symmetrical surface of $1.77 \pm 0.49$ $\mathrm{mm}$. Superpixel algorithms have been used in many areas in the literature [17-19].

In this study, various methods were used in the segmentation of high-resolution histopathological images, and their success rates are compared

This proposed study includes the method performed in part 2, the experimental results obtained within the study's scope in part 3, and the conclusion in part 4 .

\section{METHODOLOGY}

Segmentation methods used in our study are presented in this chapter. Superpixel approaches are used in various areas of image processing.

\section{A. K-Means Method}

$\mathrm{K}$-means method is used for segmentation to reduce the distance between the data and the cluster to a minimum and divide ' $\mathrm{N}$ ' data into 'c' clusters [20]. Algorithm steps are as follows;

- A random $\mathrm{k}$ number of cluster centers are determined

- The distance between cluster centers and each pixel is calculated

- The average of each cluster is calculated.

- The pixel is assigned to the closest cluster center. The process ends when the stop criteria are met. If not, the process continues from step 2 .

$b_{n}=\sum_{j=1}^{N} \max \left(d_{k-1}^{j}-\left\|x_{n}-x_{j}\right\|^{2}, 0\right)$

$\mathrm{i}=\arg b_{n}$

In Equation $1 d_{k-1}^{j}$ is the square distance between the closest cluster centers located between cluster centers. In other words, it is the cluster center of $x_{j} . b_{n}$ aims to reduce the error by placing a new cluster center at the $x_{n}$ location. $x_{j}$, are the cluster centers obtained so far [21].

In the K-means clustering algorithm, as the number of data and the number of clusters increases, the calculation time increases.

\section{B. Fuzzy C-Means Method}

The Fuzzy C-Means method is an algorithm that ensures data can be assigned to more than one cluster. With the help of its membership degree, it can have more than one cluster. The degree of membership taking values between 0 and 1 and that determines how much they join in which cluster. In the algorithm steps, first, the membership matrix $\left(\mathrm{U}^{0}\right)$ is created with random values. The threshold value $(\varepsilon)$ and the number of clusters (c) the stopping condition is determined. The value of $\mathrm{m}$, which is the fuzzy parameter, is determined. [22]

In the Fuzzy $\mathrm{C}$ Means method, the cluster center is calculated as in Equation 3. $u_{i j}$ is the membership value of the pixel in the cluster

$$
c_{j}=\frac{\sum_{i=1}^{n}\left(u_{i j}^{k}\right)^{m} y_{i}}{\sum_{i=1}^{n}\left(u_{i j}^{k}\right)^{m}}
$$

The membership matrix is calculated as in Equation 4 using $U^{k+1}$.

$$
\begin{aligned}
& u_{i j}^{k+1}=\frac{1}{\sum_{k=1}^{c}\left(\frac{d_{i j}}{d_{k j}}\right)^{\frac{2}{(m-1)}}} \\
& d_{i j}=\left\|y_{i}-c_{i}\right\|^{2}
\end{aligned}
$$

The process continues until $\max _{i j}\left\|u_{i j}^{k}-u_{i j}^{k+1}\right\|<c$ condition is met. When the process is achieved, it returns to the central matrix.

\section{Simple Linear Iterative Clustering (SLIC) Method}

Simple Linear Iterative Clustering (SLIC) is a superpixel segmentation algorithm proposed by Achanta et al. SLIC is a k-Means based algorithm that clusters neighboring pixels considering color and coordinate information [23]. When SLIC is used as a superpixel preprocessing step, it provides computational speed, memory savings [24]. The process performed by the segmentation method for each pixel is given in equation 6 .

$d_{c}=\sqrt{\left(I_{j}-I_{i}\right)^{2}+\left(a_{j}-a_{i}\right)^{2}+\left(b_{j}-b_{i}\right)^{2}}$

In Equation 7, how the coordinate information of each pixel is calculated is specified. In the equation, $\mathrm{j}$ is the pixel accepted as the center and $i$ value is the value that wants to be clustered.

$d_{s}=\sqrt{\left(x_{j}-x_{i}\right)^{2}+\left(y_{j}-y_{i}\right)^{2}}$

What is calculated in Equation 8 is the realization of assigning the relevant pixel to a center.

$D^{\prime}=\sqrt{\left(\frac{d_{c}}{N_{c}}\right)^{2}+\left(\frac{d_{s}}{N_{s}}\right)^{2}}$

The maximum expected spatial distance $\left(N_{s}\right)$ within a given cluster should correspond to the sampling interval. SLIC superpixels correspond to clusters on the lab color image plane. Determining the maximum color distance $\left(N_{c}\right)$ will not be easy as the color distances will vary significantly from image to image and cluster to cluster. This situation can be avoided by fixing $N_{c}$ 'to a fixed value of $\mathrm{m}$. 


$$
D=\sqrt{d_{c}{ }^{2}+\left(\frac{d_{s}}{s}\right)^{2} m^{2}}
$$

The $\mathrm{m}$ value in Equation 9 also determines the relative importance between color similarity and spatial proximity.

\section{Quick Shift Segmentation Method}

Quickshift is one of the popular superpixel segmentation algorithms. The principle of that algorithm is that it identifies and repeats data in a series of data points. Quickshift can be used in any field. When all data points are connected, the threshold value is used to distinguish the modes from each other. Different sets of data are separated from each other [25].

This method aims to generate superpixels by automatically changing the fast transition parameters according to a definition. Neighbours are considered within a spatial distance with a Gaussian kernel [25].

Then, as shown in the equation, it creates a tree with a higher density value that connects each image pixel $(x, y)$ to the nearest neighbour ( $\mathrm{x}$ ', $\left.\mathrm{y}^{\prime}\right)$

$\mathrm{P}\left(X_{i}\right)=\frac{1}{N} \sum_{j=1}^{N} k\left(D\left(x_{i}, x_{j}\right)\right), x_{i}, x_{j} \in R^{d}$

$\mathrm{N}$ is data and $\mathrm{k}(\mathrm{x})$ value is the kernel function with a Gaussian window. $\mathrm{D}\left(\mathrm{x}_{\mathrm{i}}, \mathrm{x}_{\mathrm{j}}\right)$ is the distance between $\mathrm{x}_{\mathrm{i}}$ and $\mathrm{x}_{\mathrm{j}}$. The distances are calculated with the equation in 11 using the Euclidean distance in the spatial and color field.

$d_{q}(i, j)=d_{x y}(i, j)+w_{q} \cdot d_{l a b}(i, j)$

The $w_{q}$ in Equation 11 is the weighting parameter, and the smaller the weighting parameter, the more critical the spatial field [26].

\section{E. Felzenszwalb Segmentation Method}

Felzenzwalb is an algorithm based on local variation graphics. Since the algorithm does not have a density limitation, it typically produces zones of irregular shapes and sizes [27]. The algorithm works almost linearly with time and is fast in practice. The critical segmentation method is ignoring the detail in high variable images while preserving low variable images [28].

Graph-based algorithm input is $\mathrm{G}=(\mathrm{V}, \mathrm{E})$ with $\mathrm{n}$ corners and $\mathrm{m}$ sides. Its output is the division of $\mathrm{V}$ into $\mathrm{S}=\left(C_{1}, C_{2}, \ldots C_{r}\right)$ components [29].

$\mathrm{C} \subseteq \mathrm{V}$ is the component's, internal difference; the maximum weight covering the component minimum is expressed as MST (C, E). So;

$\operatorname{Int}(C)=w(e)$

The difference between $C_{1}, C_{2}$ components and $\mathrm{V}$ are defined as the minimum weight edge connecting the two parts. So;

$$
\operatorname{Dif}\left(C_{1}, C_{2}\right)=w\left(v_{i}, v_{j}\right)
$$

\section{F. Watershed Segmentation Method}

Watershed is well suited for superpixel production; It provides good adherence to the object's boundaries when the image gradient is calculated and allows control by selecting the resulting images number and spatial arrangement [30]. The grayscale gradient image where it states the boundaries in the algorithm is required. It sees the bright pixels in the image as the landscape where it consists of high peaks. Then it creates a distinct image segment from high hills and flood basins [31].

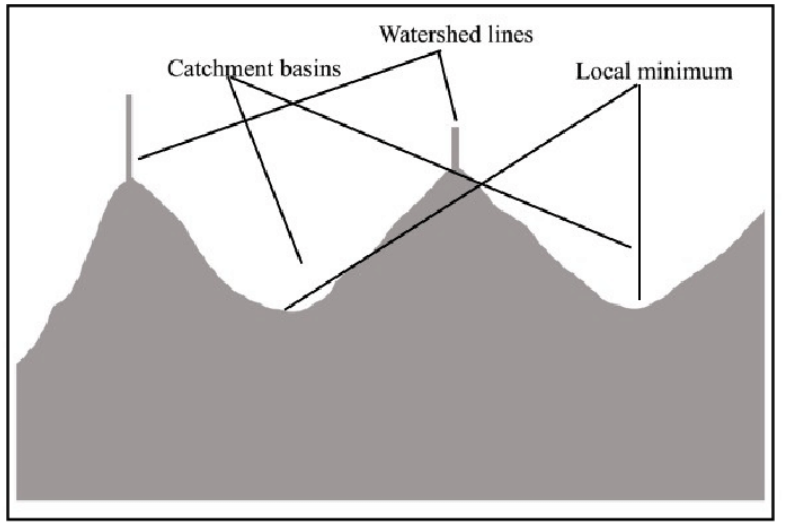

Fig.1. Modeling of the Watershed algorithm [32]

A grayscale image is defined as $\mathrm{G}=(\mathrm{D}, \mathrm{E}, \mathrm{f})$. You assume that the image $\mathrm{f}$ is the continuous space $\mathrm{C}(\mathrm{D})$, only critical points in a connected domain $\mathrm{D}$ form function on $\mathrm{D}$. The distance between points is calculated as in the following equation [33]:

$T_{f}(p, q)=i n f_{\lambda} \int_{\lambda} \| \nabla f(\lambda(s) \| d s$

\section{G. ERS Segmentation Method}

ERS segmentation is a graph-based superpixel segmentation method proposed by Lui et al. Unlike wellknown superpixels such as SLIC, and ERS attempts to find compact and homogeneous superpixels using a graph-based approach. ERS consists of two components as the entropy ratio and balancing term. Entropy ratio, tight formation, homogeneous clusters, and the balancing period that create compact appearance provide similar clusters [34]. In the ERS algorithm; Similarities between neighbouring vertices $\left(w_{i j}\right)$ are calculated, the number of clusters $(\mathrm{k})$ and the weight factor $(\lambda)$ are set. The original subset is set to $A=\emptyset$, and the edge is set to $U=E$. For each side $a \in U$, the function $F(A U$ $\{a\})-F(A)$ is calculated. It has found a value that provides the greatest success. The function continues until A becomes $\mathrm{U}=\varnothing$ independent of $\mathrm{U}$. The superpixel segmentation in the original subset (A) is created [35]. The uncertainty of the random variable is measured by entropy $(\mathrm{H})$. With the probability mass function $p(x)$, the entropy of $\mathrm{X}$, which is a discrete random variable, is calculated as in the equation [36]; 


$$
H(X)=-\sum_{x \in X} p(x) \log p(x)
$$

\section{G.Measurements Used to Evaluate Segmentation Performance}

Some measurements were used to determine the operating performance in the experiments. These are: True Positive Ratio (TPR), True Negative Ratio (TNR), Precision (P), Overlap Ratio (OR), False Positive Ratio (FPR), False Negative Ratio (FNR) are the measures used to evaluate the segmentation performance of FM [37].

\begin{tabular}{|c|c|c|}
\hline Metric & Equation & \\
\hline Precision & $\frac{T P}{T P+F P}$ & (16) \\
\hline True Positive Ratio (TPR) & $\frac{T P}{T P+F N}$ & $(17)$ \\
\hline $\begin{array}{l}\text { F- Measure } \\
(\mathrm{F}-\mathrm{M})\end{array}$ & $\frac{2 \times \text { precision } \times T P R}{\text { precision }+T P R}$ & (18) \\
\hline True Positive Ratio (TNR) & $\frac{T N}{T N+F P}$ & (19) \\
\hline $\begin{array}{l}\text { Overlop Ratio } \\
\text { (OR) }\end{array}$ & $\frac{A(S) \cap A(G)}{A(S) \cup A(G)}$ & (20) \\
\hline
\end{tabular}

In formula 16, A (S) represents the segmented image area, while A $(G)$ describes the actual image's location. The parameters specified in the formulas are true positive (TP), true negative (TN), false positive (FP), false negative (FN), precision, and recall. True positive represents the number of pixels correctly affected as positive samples in the area of cellular structure. In contrast, true negative refers to the number of pixels outside the cellular systems and are negatively affected [35].

The data set used in our study was taken from the Beck Laboratory of Harvard University. The dataset is selected from TCGA (The Cancer Genome Atlas) data containing high-resolution histopathological images of kidney carcinoma. Histopathological images had cropped $400 \times 400$ images. Expert pathologists marked cellular structures in the images, and 64 histopathological images were used.

\section{III.EXPERIMENTAL RESULTS}

Automatic detection of diseased cellular structures on high-resolution histopathological images is of great importance for cancer detection. Different segmentation methods were used for 64 histopathological images. Some of the parameters were used to evaluate the results obtained. This study evaluated the performance of using methods for automatic cell detection in high-resolution histopathological images.

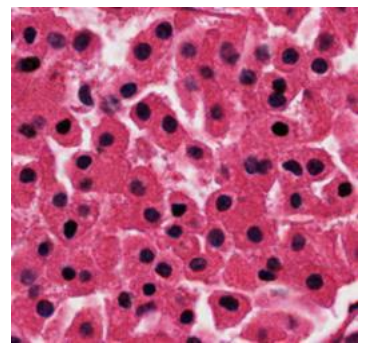

(a)

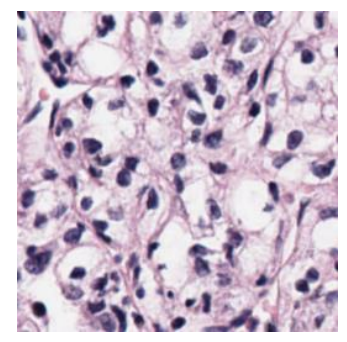

(b)

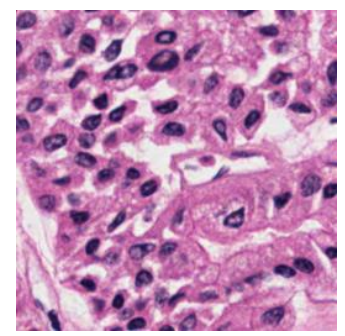

(c)

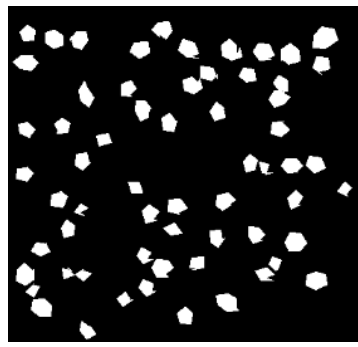

(d)

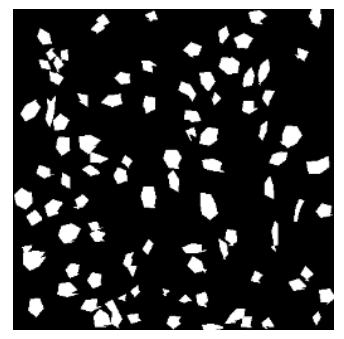

(e)

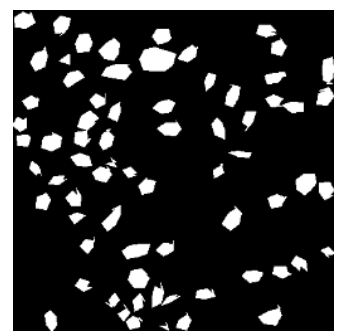

(f)
Fig.2. High-resolution images obtained in the dataset are (a), (b), and (c). The reference image of the image marked by expert pathologists are (d), (e), and (f).

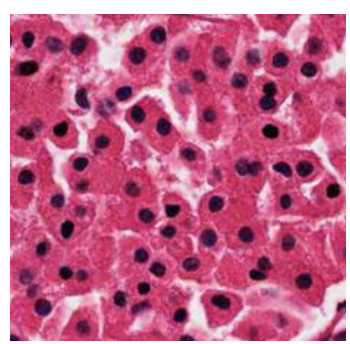

(a)

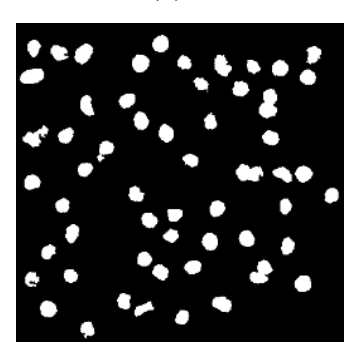

(c)

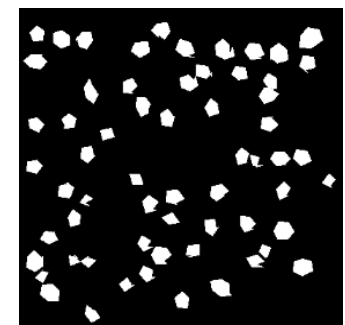

(b)
Fig.3. (a) High-resolution image obtained in the data set (b) image marked by expert pathologists (c) k-Means segmentation result

The results of k-means, fuzzy C-means, and superpixel segmentation algorithms (SLIC, Watershed, Quickshift, Felzenszwalb, ERS) were obtained on 64 high-resolution histopathological images. 
In each method, different values were given for the parameters, and the results were evaluated.

TABLE I

THE PERFORMANCE OF THE STUDIES ON THE SAME DATA SET

\begin{tabular}{|c|c|c|c|c|}
\hline Method & $\begin{array}{c}\text { True } \\
\text { Positive }\end{array}$ & Precision & $\begin{array}{c}\text { F- } \\
\text { Measure }\end{array}$ & $\begin{array}{c}\text { True } \\
\text { Negative }\end{array}$ \\
\hline KMeans & $0,60 \%$ & $0,69 \%$ & $0,61 \%$ & $\mathbf{0 , 9 7 \%}$ \\
\hline FCM & $0,60 \%$ & $0,68 \%$ & $0,61 \%$ & $\mathbf{0 , 9 7 \%}$ \\
\hline SLIC+KO & $0,69 \%$ & $0,67 \%$ & $0,63 \%$ & $0,96 \%$ \\
\hline SLIC+BCO & $0,66 \%$ & $0,68 \%$ & $0,64 \%$ & $0,96 \%$ \\
\hline SLIC-DBSCAN & $0,74 \%$ & $0,61 \%$ & $0,64 \%$ & $0,94 \%$ \\
\hline ERS & $0,65 \%$ & $0,64 \%$ & $0,61 \%$ & $0,95 \%$ \\
\hline TPRS & $0,65 \%$ & $0,63 \%$ & $0,61 \%$ & $0,96 \%$ \\
\hline SegNet & $0,70 \%$ & $\mathbf{0 , 7 1 \%}$ & $\mathbf{0 , 6 9 \%}$ & $0,95 \%$ \\
\hline Irshad vd. & $\mathbf{0 , 7 6 \%}$ & $0,62 \%$ & $0,65 \%$ & $0,96 \%$ \\
\hline Otsu & $0,67 \%$ & $0,64 \%$ & $0,62 \%$ & $0,95 \%$ \\
\hline
\end{tabular}

Table I shows the success rates of the studies belonging to the same data set. It was seen that the TPR value gave better results in FCM and K-Means methods. Quickshift, Watershed, and Felzenszwalb methods are used as different methods from the previously used dataset.

\section{Success Graphic}

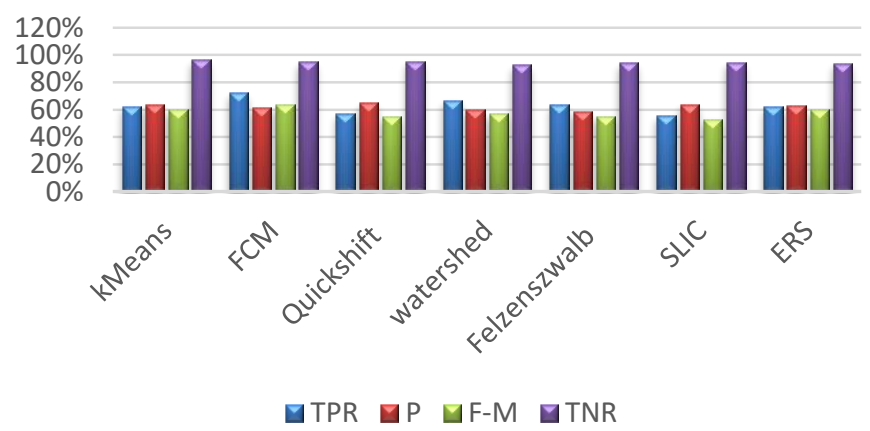

Fig.4. Performance chart of segmentation results

In Figure 4, the performance graph of segmentation algorithms used is shown.

\section{CONCLUSION}

TABLE II

PERFORMANCE OF THE USED SEGMENTATIONN ALGORITHHMS

\begin{tabular}{|c|c|c|c|c|}
\hline Method & $\begin{array}{c}\text { True } \\
\text { Positive }\end{array}$ & Precision & $\begin{array}{c}\text { F- } \\
\text { Measure }\end{array}$ & $\begin{array}{c}\text { True } \\
\text { Negative }\end{array}$ \\
\hline kMeans & $63 \%$ & $64 \%$ & $60 \%$ & $96 \%$ \\
\hline FCM & $73 \%$ & $62 \%$ & $63 \%$ & $95 \%$ \\
\hline Quickshift & $57 \%$ & $65 \%$ & $55 \%$ & $96 \%$ \\
\hline Watershed & $67 \%$ & $60 \%$ & $57 \%$ & $93 \%$ \\
\hline Felzenszwalb & $64 \%$ & $59 \%$ & $55 \%$ & $94 \%$ \\
\hline SLIC & $56 \%$ & $64 \%$ & $53 \%$ & $95 \%$ \\
\hline ERS & $64 \%$ & $63 \%$ & $60 \%$ & $94 \%$ \\
\hline \hline
\end{tabular}

As seen in Table II, seven segmentation methods were used in the study. It is seen that better success is obtained in the Watershed and FCM algorithms in high-resolution histopathological images used. Quickshift and SLIC methods gave better results in terms of precision. It is seen that there are k-Means and FCM algorithms that provide the best performance in $\mathrm{F}$ measure (F-M), and the true negative ratio is more successful in Quickshift, k-Means, and SLIC methods. Because the borders of the cells marked by the pathologists are not precisely marked, their performance decreases.

\section{REFERENCES}

[1] Anonim,2017. Erken Teşhis Hayat Kurtarır [Online] https://www.saglik.gov.tr/Eklenti/8635,kanseristatistikleridocx.docx?0

[2] A. Albayrak and G. Bilgin, 2017. Superpixel approach in high resolution histopathological image segmentation. In 2017 25th Signal Processing and Communications Applications Conference (SIU) (pp. 1-4). IEEE.

[3] J. Xu, L. Xiang, Q. Liu, H. Gilmore, J. Wu, J. Tang, and A. Madabhushi,2015. Stacked sparse autoencoder (SSAE) for nuclei detection on breast cancer histopathology images. IEEE transactions on medical imaging, 35(1), 119-130.

[4] B. H. Aksebzeci, and Ö. Kayaalti, 2017. Computer-aided classification of breast cancer histopathological images. In 2017 Medical Technologies National Congress (TIPTEKNO) (pp. 1-4). IEEE.

[5] A. Albayrak, and G. Bilgin, (2018). Segmentation of cellular structures with encoder-decoder-based deep learning algorithm in histopathological images. In 2018 Medical Technologies National Congress (TIPTEKNO) (pp. 1-4). IEEE.

[6] A.Albayrak, A. Ünlü, N. Çalık, G. Bilgin İ. Türkmen A. Çakı., ... and L. D. Ata, (2017). Segmentation of precursor lesions in cervical cancer using convolutional neural networks. In 2017 25th Signal Processing and Communications Applications Conference (SIU) (pp. 1-4). IEEE.

[7] S. Turan, and G. Bilgin, (2019). Semantic nuclei segmentation with deep learning on breast pathology images. In 2019 Scientific Meeting on Electrical-Electronics \& Biomedical Engineering and Computer Science (EBBT) (pp. 1-4). IEEE.

[8] B. Li, W. J. Niessen, S. Klein, M. de Groot, M.A. Ikram, M. W.Vernooij, and E. E. Bron,(2019). A hybrid deep learning framework for integrated segmentation and registration: Evaluation on longitudinal white matter tract changes. In International Conference on Medical Image Computing and Computer-Assisted Intervention (pp. 645-653). Springer, Cham.

[9] O. Ronneberger, P. Fischer, and T. Brox (2015). U-net: Convolutional networks for biomedical image segmentation. In International Conference on Medical image computing and computerassisted intervention (pp. 234-241). Springer, Cham.

[10] M. E. Bagdigen, and G. Bilgin, (2019). Detection and Grading of Breast Cancer via Spatial Features in Histopathological Images. In 2019 Medical Technologies Congress (TIPTEKNO) (pp. 1-4). IEEE.

[11] A. Feng-Ping, and L. Zhi-Wen, (2019). Medical image segmentation algorithm based on feedback mechanism convolutional neural network. Biomedical Signal Processing and Control, 53, 101589.

[12] K. Xia, H. Yin, P. Qian, Y. Jiang, and S. Wang, (2019). Liver semantic segmentation algorithm based on improved deep adversarial networks in a combination of weighted loss function on abdominal CT images. IEEE Access, 7, 96349-96358.

[13] Y. Feng, H. Zhao, X. Li, X. Zhang, and H. Li, (2017). A multi-scale 3D Otsu thresholding algorithm for medical image segmentation. Digital Signal Processing, 60, 186-199.

[14] T. H. Farag, W. A. Hassan, H. A. Ayad, A.S AlBahussain, U. A. Badawi, and M. K. Alsmadi, (2017). Extended absolute fuzzy connectedness segmentation algorithm utilizing region and boundary- 
based information. Arabian Journal for Science and Engineering, 42(8), 3573-3583.

[15] I. F. Salazar-Reque, S. G. Huamán, G. Kemper, J. Telles, and D. Diaz, (2019). An algorithm for plant disease visual symptom detection in digital images based on superpixels. Int. J. Adv. Sci. Eng. Inf. Technol, 9(1), 194-203.

[16] W. Qin, J. Wu , F.Han, Y. Yuan, W. Zhao, B. Ibragimov, ... and L. Xing, (2018). Superpixel-based and boundary-sensitive convolutional neural network for automated liver segmentation. Physics in Medicine \& Biology, 63(9), 095017.

[17] O. F. Kar, A. Güngör, S. Ilbey, and H. E. Güven, (2018, May). An efficient parallel algorithm for single-pixel and fpa imaging. In Computational Imaging III (Vol. 10669, p. 106690J). International Society for Optics and Photonics.

[18] H. Yu, M. Jiang, H. Chen, J. Feng, Y. Wang, and Y. Lu, (2017). Super-pixel algorithm and group sparsity regularization method for compressed sensing MR image reconstruction. Optik, 140, 392-404.

[19] S. Kaur, R. K. Bansal, M. Mittal, L. M Goyal, I. Kaur, and A. Verma, (2019). Mixed pixel decomposition based on extended fuzzy clustering for single spectral value remote sensing images. Journal of the Indian Society of Remote Sensing, 47(3), 427-437.

[20] T. Kanungo, Mount, DM, Netanyahu, NS, Piatko, CD, Silverman, R., and Wu, AY (2002). An effective k-mean clustering algorithm: Analysis and application. IEEE processes on model analysis and machine intelligence, 24 (7), 881-892.

[21] A. Likas, N. Vlassis, and J. J. Verbeek, (2003). The global k-means clustering algorithm. Pattern recognition, 36(2), 451-461.

[22] B. K. Tripathy, A. Basu, and S. Govel, (2014). Image segmentation using spatial intuitionistic fuzzy $\mathrm{C}$ means clustering. In 2014 IEEE International Conference on Computational Intelligence and Computing Research (pp. 1-5). IEEE.

[23] S. Zhang, Z. Ma, G. Zhang, T. Lei, R. Zhang, and Y. Cui (2020). Semantic Image Segmentation with Deep Convolutional Neural Networks and Quick Shift. Symmetry, 12(3), 427.

[24] R. Achanta, A. Shaji, K. Smith, A. Lucchi, P. Fua, and S. Süsstrunk, (2012). SLIC superpixels compared to state-of-the-art superpixel methods. IEEE transactions on pattern analysis and machine intelligence, 34(11), 2274-2282.

[25] S. Zhang, Z. Ma, G. Zhang, T. Lei, R. Zhang, and Y. Cui, (2020). Semantic Image Segmentation with Deep Convolutional Neural Networks and Quick Shift. Symmetry, 12(3), 427.

[26] F. M. Osman, and M. H. Yap, (2020). Adjusted Quick Shift Phase Preserving Dynamic Range Compression method for breast lesions segmentation. Informatics in Medicine Unlocked, 100344.

[27] A. Levinshtein, A. Stere, K. N. Kutulakos, D. J. Fleet, S. J. Dickinson, and K. Siddiqi, (2009). Turbopixels: Fast superpixels using geometric flows. IEEE transactions on pattern analysis and machine intelligence, 31(12), 2290-2297.

[28] P. F. Felzenszwalb, and D.P. Huttenlocher, (2004). Efficient graphbased image segmentation. International journal of computer vision, 59(2), 167-181.

[29] L. Yao, and S. Muhammad, (2019). A novel technique for analysing histogram equalized medical images using superpixels. Computer Assisted Surgery, 24(sup1), 53-61.

[30] [30] V. Machairas, E. Decencière, and T. Walter, (2014). Waterpixels: Superpixels based on the watershed transformation. In 2014 IEEE International Conference on Image Processing (ICIP) (pp. 4343-4347)

[31] [31] J. Schönberger, A. Siqueira, A. Mueller, E. Gouillart, G. Lee, M. Harfouche, J. Warner, J.N. Iglesias, L. Grüter, M. Corvellec, R. Fezzani, F. Boulogne, E. Panfilov, and S. Walt, (2014) https://scikitimage.org/ [Online]

[32] [32] Benson, CC, V. Deepa, Lajish, VL, and K. Rajamani, (2016). Brain tumor segmentation from MR brain images using advanced fuzzy c-averaged clustering and watershed algorithm. In the 2016 International Conference on Informatics, Communication and Informatics Advances (ICACCI) (p.187-192).
[33] J. B. Roerdink and A. Meijster,(2000). The watershed transform: Definitions, algorithms and parallelization strategies. Fundamenta Informatica, 41(1, 2), 187-228

[34] A.Albayrak, and G. Bilgin, (2019). Automatic cell segmentation in histopathological images via two-staged superpixel-based algorithms. Medical \& biological engineering \& computing, 57(3), 653-665.

[35] W. Wang, D. Xiang, Y. Ban, J. Zhang, and J. Wan, (2017). Superpixel segmentation of polarimetric SAR images based on integrated distance measure and entropy rate method. IEEE Journal of Selected Topics in Applied Earth Observations and Remote Sensing, 10(9), 4045-4058.

[36] M. Y. Liu, O. Tuzel, S. Ramalingam, and R. Chellappa, (2011). Entropy rate superpixel segmentation. In CVPR 2011 (pp. 20972104). IEEE.

[37] A. S. Glas, J. G. Lijmer, M. H. Prins, G. J. Bonsel, and P. M. Bossuyt, (2003). The diagnostic odds ratio: a single indicator of test performance. Journal of clinical epidemiology, 56(11), 1129-1135.

\section{BIOGRAPHIES}

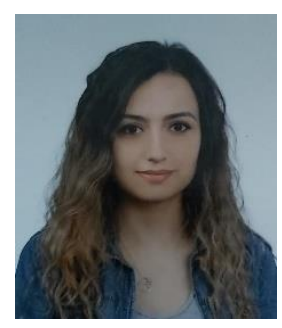

GAMZE MENDi received the B.S. degree in electrical and electronics engineering from Siirt University, Siirt, Turkey in 2017. She received the M.S in electrical and electronics engineering from Batman University Batman, Turkey in 2021. I am working as an engineer in DEDAS since 2018

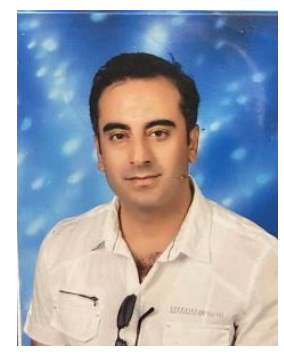

CAFER BUDAK received his B.Sc. and M.Sc. degrees in the department of Electrical Electronics Engineering from DicleUniversity in 1998 and 2008, respectively. $\mathrm{He}$ is received his $\mathrm{PhD}$ degree in the department of Electrical Electronics Engineering from Firat University in 2014. He is currently an Assistant Professor at the department of Biomedical Engineering at the Dicle University in Turkey. His research interests include artificial intelligence, deep learning, machine learning, image processing, Graph Neural Network, and histopathological images 